\title{
A Spectrum of Audience Interactivity for Entertainment Domains
}

\author{
Alina Striner ${ }^{1(\otimes)}$, Sasha Azad ${ }^{2}$, and Chris Martens ${ }^{2}$ \\ 1 Centrum Wiskunde and Informatica (CWI), Amsterdam, Netherlands \\ alina.striner@gmail.com \\ 2 North Carolina State University, Raleigh, NC 27695, USA
}

\begin{abstract}
The concept of audience interactivity has been rediscovered across many domains of storytelling and entertainment-e.g. digital games, in-person role-playing, film, theater performance, music, and theme parks - that enrich the form with new idioms, language, and practices. In this paper, we introduce a Spectrum of Audience Interactivity that establishes a common vocabulary for the design space across entertainment domains. Our spectrum expands on an early vocabulary conceptualized through co-design sessions for interactive musical performances. We conduct a cross-disciplinary literature review to evaluate and iterate upon this vocabulary, using our findings to develop our validated spectrum.
\end{abstract}

Keywords: Audience interaction · Audience participation ·

Entertainment $\cdot$ Agency $\cdot$ Performance interaction $\cdot$ Immersion

\section{Introduction}

Interactivity has the power to immerse and empower audiences across divergent domains. Although these mediums use different terminology, sometimes describing interactive approaches as participatory or immersive, their desired outcome is to design fulfilling storytelling experiences. In Hamlet on the Holodeck, for instance, Murray argues that future science fiction authors will be challenged to define rules for narrative interaction that transform passive readers into audiences engaged in immersive and reactive narrative experiences [81].

In pursuit of this dream of the Holodeck, HCI research often designs novel technology to support immersive experiences $[65,105]$. However, generalizing and characterizing rules for interaction is as tricky for writers and designers as it is for practitioners [20]. Designing interactive experiences often means learning from previous work and building experiences using available tools. Since interactive audience experiences exist in a range of contexts, designers are often limited to learning from their area of expertise. We posit that in addition to new technology, the HCI community needs conceptual tools that help designers across performance mediums consider and compare how audiences can interact. 
To develop new forms of artistic expression, HCI practitioners require a common language to compare and learn from diverse experiences. Prior work defined models that broadly measure [119], and describe audience agency and participation $[9,33,90,107,126]$, but literature suggests that more complicated relationships must be defined to address Murray's fully interactive world [81].

This paper expands on an early spectrum conceptualized through co-design sessions for interactive musical performances [108], using it to develop our Common Spectrum of Audience Interactivity for Entertainment Domains. Our approach explicitly allows designers across domains to discuss interactive experiences using a common taxonomy. First, we define audience interactivity, describe its benefits, and overview previous efforts to characterize interactivity. Then, we conduct an extensive review of interactive experiences across theater, theme parks, and games, three domains that represent diverse audiences, modes of interaction, and performance spaces. Our findings validate and expand on the early spectrum, refining it with additional levels, labels, and definitions. For clarity, the paper presents the literature review after introducing the new spectrum.

In summary, our work (1) overviews previous work on audience interactivity, (2) reviews literature across three entertainment domains, and (3) presents a new Spectrum of Audience Interactivity.

\section{Related Work}

In this section, we first describe how storytelling has evolved to include audiences, resulting in more immersive and engaging experiences. Then, we define interactivity as audience agency and participation in performance, and describe how it contributes to immersion and engagement. Finally, we overview previous efforts to characterize audience interactivity.

\section{$2.1 \quad$ Storytelling}

Throughout history, narratives have defined human culture and entertainment, transporting audiences [48] by creating "an experience of cognitive, emotional, and imagery involvement." In our research, we use Zimmerman's definition of narrative [126], building on Miller [78], who defines narrative as an initial state, a change in that state, and insight brought about by that change. We also adopt the term transmedia $[27,44]$ to refer to interactive audience experiences.

In transmedia experiences, narratives invite audiences to interact with experiences. Theme parks fulfill audience needs to interact by creating a fantasy of another place and time [24,79]. Purposely designed to be isolated, theme parks invite guests to travel [29], to transport themselves to a new location. Leaving the real world at the parking lot, guests gain temporary "citizenship" to a fantasy world [17], escaping the rules and conventions of the outside world [118] for one with no clocks [24] or defined social barriers [12].

We see this model replicated in live theater. In audience-driven experiences like Coffee! A Misunderstanding [105], authors invite audiences to change the 
direction of an improvised narrative. Other examples include The Night of January 16th [91], in which audience members play the role of a courtroom jury, and Drood [89], a musical adaptation of a murder mystery. Games likewise offer players roles in predefined narratives, or allow narratives to naturally emerge from play [70,94], such as in the interactive drama Façade [69], where virtual characters respond to a player-performer narrative.

\subsection{Defining Audience Interactivity}

The role of the audience has changed. The capacity to alter and transform experiences has empowered audiences [75], leading to a dissolution of traditional audiencehood [16]. Previous work has described degrees of audience immersion in a narrative, however, the relationship between immersion, audience, and performers have not yet been explored. This paper extends current definitions to concretely classify the full breadth of audience experiences in entertainment.

For this reason, we define an audience member broadly; as a bystander, spectator, customer, participant, or player. Likewise, we define audience interactivity as a range of experiences that may allow audiences to participate or interact. These experiences may vary in:

- Physical and Virtual Mediums. Experiences can be physical, such as live theater, or virtual, such as VR or Twitch streams.

- Location. Experiences settings may vary in size and scale, from a single room (or virtual dungeon), to a university campus (or virtual world).

- Formality and Setting. Experiences can be private or public. For instance, an arcade simulator may be a individual experience, a murder mystery might include a group of friends, and a street performance may be public-facing.

- Ratio of Participants to Performers. Experiences may have varied ratios of audiences to performers. For instance, a massively-multiplayer role-playing game (MMORPG) may have thousands of players, while an interactive art installation may have no designated performers.

- Audience Influence and Agency. Experiences may afford audiences a range of influence or agency. For instance, a formal theater might designate when audiences should interact in performance, whereas a street performance might give them the freedom to join in when they feel comfortable.

- Tools \& Technology. Interactive experiences use a range of tools to create interaction. Tools can range from physical props and costumes to smartphones, tablets, or wearables.

\subsection{Supporting Engagement and Immersion}

Entertainment literature supports the value of audience interaction to create engagement and immersion [111]. For instance, Green et al. found that narrative transportation can affect persuasion and belief change, as well as enjoyment [48].

Engagement refers to the intensity and emotional quality of user involvement [43]; engaged users exhibit positive emotion, and show sustained cognitive 
task involvement [41]. Engagement is often created through immersion [15], a feeling of "deep play" [26,32] that furthers emotional investment [111]. Several constructs [47] have been proposed to describe immersion. Ermi and Mayra [32] divide immersion into sensory immersion, challenge-based immersion, and imaginative immersion. Brockmyer [15] suggests that sensory immersion often creates a sense of presence or "being there," surrounded by another reality that takes over attention and perception $[26,32,74]$. Likewise, Csikszentmihalyi describes the pinnacle of challenge-based immersion as flow $[25,31,58,99]$, a state of total task absorption and optimal performance [32].

\subsection{Previous Efforts to Describe Interactivity}

Previous research endeavored to characterize interactivity in media experiences. Relatively simple models include Everett's single-dimensional scale that rated the interactivity of communication technologies [33], and Rafaeli [90], who classified media based on audience responsiveness. Based on empirical data from questionnaires answered by 6700 players, Yee [124] added an "immersionist" factor to Bartle's classification of players into achievers, explorers, socializers and killers [7]. Zimmerman [126] identified four modes of audience interactivity that complement our goal of broadly defining a taxonomy; Cognitive Interactivity, a response to an internalization of a narrative, Functional Interactivity, interaction with physical text such as turning pages, Explicit Interactivity, participation in narrative flow by making choices and participating in narrative events, and Metainteractivity, interaction that allows for narrative construction, deconstruction, and reconstruction.

\section{Spectrum of Interactivity for Music (Striner \& McNally 2017)}

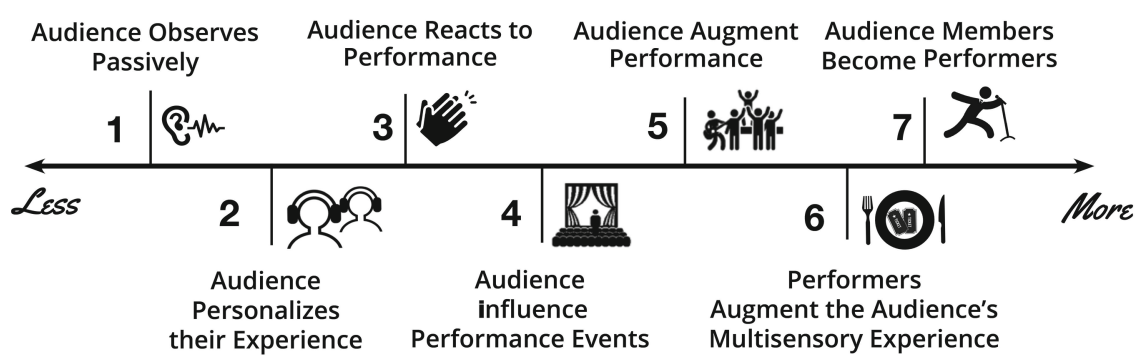

Fig. 1. Early spectrum of interactivity for musical performances [108]

Multiple models characterize interactivity by the choices and actions of audiences [45,61-63,103]. Lindley compared audience types, motivations, and play styles across current literature [88,121], and defined his own taxonomy [64] to describe three attitudes in narrative; the audience, the performer, and the immersionist. Steuer [107] expanded on Everett's characterization of interactivity with a two-dimensional model based on vividness, the richness of a mediated 
environment, and interactivity, a user's ability to modify the vividness of their experience. While Steuer's method is highly cited as a measure of immersion and engagement, it notably fails to provide explicit criteria to map new experiences onto his scale [55]. Laurel's three-dimensional model further characterizes interactivity by frequency, the range of choices available, and the extent to which choices affect experience [62]. Likewise, Goertz introduced a four dimension scale of interactivity using degrees, numbers, and flexibility of choice $[45,55]$. We extend these models, accounting for both audience engagement and agency.

Interactivity has also been discussed in great detail by researchers in HCI [61,101]. Zeltzer describes autonomy and interaction as a single dimension that encompasses all aspects of an audience's relationship to their environment [125]. Laurel further emphasizes the experiential nature of interaction with media technologies [61]. Both [61] and [107] describe media use in terms of mimesis, likening the relationship between users and technology to actions in a play, encouraging users to develop a first-person, not third-person, relationship with their environment. Engagement, which Laurel (1991) describes as a primarily emotional state with cognitive components [63], serves as a critical factor in arousing a feeling of "first-personness" [107].

Previous work by Striner and McNally [108] stewarded a first step toward understanding the many ways in which technology can allow audiences to interact with musical performances. Their work developed a spectrum of interactivity (Fig. 1) for musical performances from children's codesign sessions using Cooperative Inquiry (CI) derived from Participatory Design $[28,50]$. Using their spectrum as a starting point, we conducted an extensive, cross-disciplinary literature review to evaluate and iterate upon this vocabulary. This paper presents findings from the literature survey and a revised spectrum of interactivity.

\section{Method}

The goal of this work is to develop a taxonomy of audience interactivity to facilitate communication and collaboration among experts and designers in a wide variety of entertainment domains. This spectrum enables designers and practitioners across domains to discuss and learn from a broad range of experiences, and to consider challenges inherent to diverse audience interactivity designs. Building on prior work [108], this research evaluates and generalizes findings from music across various entertainment domains through a comprehensive review of audience interactivity literature in theater, theme parks, and games and introduces a common Spectrum of Audience Interactivity for entertainment. In this section, we first overview the underlying factors for our choice of theater, theme parks, and games as our three representative entertainment domains. Then, we describe our literature review process.

\subsection{Choice of Entertainment Domains}

Audience interactivity exists across a broad range of entertainment domains [40, $42,86,100]$. To validate Striner's spectrum [108], we considered how well it 
Table 1. Index of literature review organized by theory, storytelling, theater and music, theme parks, games, and transmedia topics.

\begin{tabular}{|c|c|}
\hline Topic & Citation index \\
\hline Theory & $\begin{array}{l}8,9,12,13,14,15,18,21,22,25,28,31,32,33,37,41,42,45,46,49 \\
50,52,55,60,61,64,68,74,75,78,81,90,99,101,108,109,126\end{array}$ \\
\hline Storytelling & $\begin{array}{l}5,9,19,20,22,43,44,48,49,54,63,67,70,76,81,84,86,91,93,114, \\
116,120,127\end{array}$ \\
\hline Theater, music & $\begin{array}{l}1,8,10,11,12,16,23,30,34,38,40,54,56,57,60,62,69,71,76,80, \\
82,83,84,88,89,92,96,105,107,109,110,117,121,123\end{array}$ \\
\hline Theme parks & $2,17,24,29,35,51,59,65,72,79,85,95,98,103,119$ \\
\hline Games & $\begin{array}{l}3,4,5,7,8,10,15,18,32,36,39,41,47,53,58,66,70,74,94,97,100, \\
104,111,112,113,114,115,118,125,127\end{array}$ \\
\hline Transmedia & $9,18,20,27,30,39,44,73,77,87,92,102,105,107,117,122$ \\
\hline
\end{tabular}

reflected interactivity across three domains - theater, games, and theme parks that embodied the range of audience interaction described above. Together, our review uncovers insights that inform our iteration on the spectrum.

The three domains vary greatly in form. Theater and music performances are primarily physical experiences that occur in dedicated venues. Conventional theatrical segregates audiences from performers, curbing feedback to pre-and-post show clapping and cheering [60], while contemporary theater allows audiences to contribute to performance, encouraging spontaneous [68] and structured participation $[96,105]$. In contrast, games exist in a range of physical and virtual forms, from tabletop games that build narrative through a shared imaginative fantasy [36], to video games that immerse audiences through integrated graphics, animation, and reward structures [104,111]. In juxtaposition to theater and games, theme parks created shared experiences for divergent audiences. Based on ancient and medieval religious festivals, trade fairs, and traditional amusement parks [79], themes parks assimilate storytelling [17,95], simulation, and interactivity $[79,98]$ through blended physical and virtual experiences.

\subsection{Literature Review Process}

The primary goal of this work was to understand how the three representative domains describe audience interactivity. Our goal was to understand what interactions existed in those domains.

We extensively reviewed literature on interactive audience experiences across academic publications and in practitioner mediums. We systematically reviewed multiple databases (e.g. AAAI, ACM, PsycINFO, CiteSeerX, CogPrints Electronic Archive, ResearchGate, TRLN) for a range of topics (previous definitions and models of audience interactivity, engagement, immersion, agency, mediums of interaction, and roles), performing "related article" searches to identify model applications and limitations. Next, we shortlisted articles that defined interactivity or described interactive experiences in the three domains. In parallel, we came 
up with a list of synonymous phrases and keywords across the three domains, and searched websites and blog posts for descriptions of practitioner experiences. We analyzed domain publications to understand how the original spectrum levels were reflected in academic literature, and to identify gaps where literature did not fit the original spectrum. When domains were not evenly represented at a level, we performed a secondary Google Scholar search to identify any literature we may have missed. The literature we reviewed is indexed by topic in Table 1 .

\section{Summary of Results}

This section summarizes our literature findings and introduces our Spectrum of Audience Interactivity for Entertainment Domains. First, we affirm the presence of a spectrum, describe modified levels, and present our validated spectrum.

\subsection{Confirming the Existence of a Spectrum}

The literature review affirmed the presence of the interactivity continuum, finding that interactivity ranged from passive to active experiences delineated by the agency of individual audience members. "Passive" and "personalized experiences" gave audiences agency over themselves, and "influencing," "augmenting," and "becoming a performer" levels gave audiences agency over other audience members, performers, and over the larger experience. Cross-domain literature supported the presence of these different levels, however we found that interactivity was more prominent in some domains; for instance, theater and music predominantly use interactivity to influence and augment performances [105,116,123], games employ audiences as performers [70,97], and theme parks create personalized and bidirectional experiences [95, 118].

\subsection{Modified Levels}

Our review found that the spectrum required some modification. Shown in Fig. 2, the new spectrum introduces a new level of audience interactivity and modifies the name of an existing level.

Bidirectional Influence. The early spectrum included the level "Performers Augmenting the Audience's Multisensory Experience." This level was difficult to describe, however, we found that "Bidirectional Influence" clearly characterized the back-and-forth dynamic of interactive performance.

Take over Performance. The early spectrum described "Become Performers" as the highest level of interactivity. However, we found that interactivity extended beyond this; audience members could not only become performers, but fully control an experience. For instance, audience members invited into a drum circle could lead the music. Thus, we added a new level, "Take over the Performance," that describes this experience. 


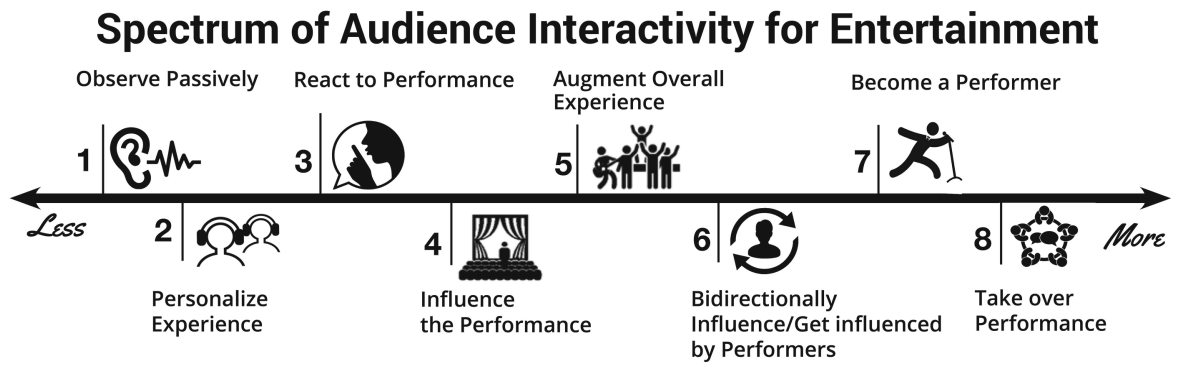

Fig. 2. The new Spectrum of Audience Interactivity for Entertainment Domains. We map audience interactivity from left to right; from least to most active.

\subsection{Proposed Spectrum of Audience Interactivity}

Presented in Fig. 2, the Spectrum of Interactivity for Entertainment Domains expands on Striner's Spectrum of Interactivity [108] using findings from the literature. Least interactive on the spectrum are (1) observing passively, referring to an audience member cognitively shaping their experience, and (2) personalizing their experience. More interactive is (3) reacting to performance, a level that describes how audience members react to performance and to one another, such as by clapping or responding to a comment on YouTube.

In (4) audience members influence the performance, exerting indirect control over the overall experience. For example, virtual audiences watching a Twitch stream could suggest a way for a streamer to solve a puzzle. Audience members in (5) augment the overall performance experience without explicitly becoming performers, for instance, dancing along at a rock concert. In (6) bidirectional influence between audience and performers, performers explicitly respond to the audience's influence or reactions, such as Mickey Mouse waving back at children.

Higher levels give audience members an explicit role in the performance, allowing them to (7) become performers and (8) take over the performance. In the former, performers are in control, for instance, audiences singing along with a choir, while in the latter, audiences take control. For example, an audience member invited to perform karaoke onstage would take over a performance.

\section{$5 \quad$ Review of Interactivity Levels}

The following section presents our review of the interactivity literature, organized from least to most interactive across the levels of our proposed Spectrum.

\subsection{Interactivity in Passive Experiences}

Traditional performances assume a clear distinction between the role of the audience and performers [16]: audiences do not interact with performers or have a role in the direction of performance or narrative. Forlizzi and others $[37,57,126]$ 
contradict this assumption, suggesting that audiences can interact with experiences cognitively, through a psychological reader-response that imbues seemingly passive experiences with an abundance of emotional interaction.

The literature suggests that audiences participate in collective emotional experiences such as laughing or holding their breath that validate their personal experiences; this helps explain why the presence of an audience is essential to the sense of "liveness" [92]. HCI research has studied passive engagement by watching audience expressions and analyzing gestures using computer vision [14,73].

Research also argues that audience interaction is not always necessary or appropriate [108]. Green et al. [49] discuss how participants may simply wish to be distracted or passively entertained [14] by fiction. This outcome is further supported by literature on interactive film suggesting that passive experiences allow audiences to absorb, appreciate, and reflect on performance $[14,48,120]$.

\subsection{Interactivity Through Personalization}

Personalization in interactivity describes the task of tailoring experiences to audience preferences, tastes, or capabilities. Theme parks fully embrace personalization in order to fully immerse audiences in fantastical worlds [72]; guests can meet characters [53], and personal experience narratives [24,95]. Paralleling these physical experiences, recent advances in narrative intelligence and augmented and mixed reality have likewise allowed for games to be personalized to player locations [4,66], abilities [97,113], and preferences [110].

Stapleton [106] describes how audiences personalize performances, discussing how a story originating in print (e.g. Harry Potter) can ignite a surge in new markets in games, theme parks, and costumes. Using dress to personalize experiences [108] is heavily paralleled in literature; Eicher's theory describes dressing up in fantasy costumes as a communication of the secret self, where the bulk of fantasy interactions takes place $[30,39]$. Similarly, Miller proposes a construct of fantastic socialization, where individuals play unrealized roles "constructed only with the cooperative help... and the contrasting foil provided by others" [46,78]. Fron et al. define such personalization as a co-performative act with other spectators, gaining pleasure from the ingenuity and artistry that go into creating one's persona and costume $[8,39,53]$. This style of personalization can be seen at American cultural festivals such as DragonCon [39], and also reflects Zimmerman's "meta-interactivity" mode [126].

\subsection{Reacting to the Performance}

Reacting to performance is a staple of traditional audience experience [60]. Literature suggests that audience members enhance the collective audience experience by influencing others' reactions [87]; Brignull and Rogers [13] explain that such interactions begin with peripheral awareness, transition to focal awareness, and culminate in direct interaction with the display. Their research observes the "honey pot" effect, in which bystanders are more likely to cross interaction 
thresholds when others do. For instance, audiences are likely to give a standing ovation (or throw rotten fruit) when others do the same [56]. An immersive interactive play, Sleep No More [116], extended this concept, allowing live and remote audiences to communicate through Internet-of-things (IoT) props.

Theme park literature characterizes this phenomenon as a learning tool. For instance, guests at the Wizarding World of Harry Potter watch others learn the mechanics of "casting a spell" $[18,59]$. Reeves describes this experience as an entertainment and teaching experience [92] that allows audiences to study interaction while waiting their turn. Magic Kingdom line experiences actively design for this affordance; guests in line for a Peter Pan ride view members ahead of them play with interactive shadow puppet displays, ringing bells, or even releasing Tinker Bell from inside a lantern [3,35]. This, in turn, prompts them to interact, mimicking scenes they have seen before, playing on each other's interactions and inventing new ones. Michelis [77] describes this phenomenon of the phases of interactions with gesture-based displays as an "audience funnel."

\subsection{Influencing Performers}

Interaction often allows audience members to indirectly influence the performance experience. Influencing performance includes visual voting systems [117], and audience input in improve [76]. While these types of interactions are popular, theater literature suggests that they are often asynchronous or inequitable [60], prioritizing audience members closer to the stage [23] or in positions of power [80].

Technical advancements have helped support democratic influence over voting. In an early example, audiences at the 1967 World's Fair in Montreal voted on alternative endings to a film [2]. Likewise, technology has allowed audiences to influence narratives [19], dialogues [105], or musical compositions [38]. Literature also found that designers wanted audiences to influence different sensory modalities, such as controlling gusts of wind onstage [108].

\subsection{Augmenting the Experience}

The literature suggests that audiences also want to augment experiences [108]. One way to do this is through multisensory design. For instance, child codesigners augmented music experiences with tangible "sound chips" [108]. Relatedly, Stapleton and Hughes [106] found that immersing movie-goers in multisensory mixed reality trailers created fond memories and positive associations.

Literature suggests that audiences can likewise augment experiences by adopting a composition role. Winkler notes that interactive computer music can "create new musical relationships" between audience and performers [123]; for instance, McAllister [71] allowed audience members to add to a digital score synced to a real-time display for musicians to read. Likewise, audiences can "compose" by dancing to music during performances [83,109]. 
This compositional relationship between audience and performers can also be asynchronous; for instance, van Troyer [115] introduced an interface for audiences to co-create asynchronously with composers by drawing "constellation" maps that synthesized new music from previous pieces. Similar examples exist in interactive fiction design. For instance, Machado [67] recounts a storytelling environment, Once Upon A Time, that developed characters, story themes, and narratives out of interactions with children.

\subsection{Bidirectional Influence}

Both physical and digital interactive performances lean heavily on the affordances of bidirectional interaction. For instance, gospel music uses call-andresponse to nudge democratic audience participation [82], and computational narratives personalize player experiences by iteratively tracking and adapting narrative scheduling to player pacing [6]. Similar research has produced a virtual dance partner that improvises dance moves based on audience actions [54], and a narrative agent that responds to audience gestures with dialogue [84].

As well as responding to each other, some literature characterizes bidirectional interactions as "pushing and pulling" between audiences and performers. For instance, Rickman [93] described a text narrative mechanic that drives the narrative forward by using word selection to reveal additional information about an object or action [22]. Curiously, the research suggests that bidirectionality many not always be intentional. For instance, Van Maanen [118] describes how at Walt Disney World, guests and cast members cyclically affect each other; cast members are required to smile, but guests not smiling can ruin an operator's day.

\subsection{Becoming Performers}

All three domains allow audience members to take on performative roles, but differ in their approach. Games create immersion by giving players a sense of control [21], allowing users to select strategies, and affect outcomes [97]. Video games have an inherent performative experience, allowing audiences to dually function as players and audiences members [104], imbuing players with spectatorship in-between moments of play [112]. For instance, LARPS (Live-actionrole-playing games) are considered performance-play experiences [102]. LARPS have no separate audience members, allowing audiences to extemporaneously create engaging narratives from limited preparatory materials [102].

Fantasy sports games further blend the roles of audiences and performers [100] by integrating the "activity of a virtual game and spectatorship of a real sport" [100]; Developments in large-scale streaming, tangible interfaces, and virtual and augmented reality have further changed the game viewer landscape. Twitch allows audiences to watch, and interact with streamers during games [114]. Similarly, augmented reality has given players and viewers a way to experience narratives in physical space $[5,51,106]$. 
Although less accessible than games [24], theme parks fully embrace audiences in performative roles, integrating storytelling [17,95], simulation, and interactivity [79,98], and emphasizing physical experiences. Theme park experiences often give audiences a chance to re-experience character roles and narratives. These firsthand narratives lean heavily on multisensory, spatial, and temporal experiences [79] to create a sense of presence $[17,85]$.

\subsection{Taking over Performance}

Performance experiences also allow audiences to "take over" performances, building self-esteem [83] by allowing audiences to reshape existing experiences or cocreate new ones. For instance, Boal [11] developed the Theater of the Oppressed to promote social and political change; audience members became "spectactors," who used the medium to explore, and analyze their personal experiences. Likewise, home experiences like Guitar Hero [10] and Hyperscore [34] have contributed to music appreciation by bridging skill gaps.

Relatedly, music experiences help audiences make sense of and appreciate complex arts [82] by allowing them to co-create new experiences. For instance, Whitacre [122] developed a virtual choir that allowed singers all over the world to contribute to a performance, and Machover's City Symphonies [52] allowed audiences to contribute ambient sounds that made up their city.

Notably, in theater, the role of audiences as a performative agent is contested. In Hamlet on the Holodeck, Murray [81] suggests that audience participation may be "awkward" and potentially "destructive;" she describes a Woody Allen story, the Kugelmass Episode [1] where a literature professor jumps into the pages of Madame Bovary, only to confuse the narrative of the novel; "Who is this character on page 100? A bald Jew is Kissing Mme Bovary?" With this, Murray points out that "when we enter the enchanted world as our actual selves, we risk draining it of its delicious otherness" [81].

\section{Conclusion}

The goal of this work was to develop a taxonomy to explicitly characterize how audiences can interact and influence experiences across a range of entertainment domains. The spectrum aims to be a useful resource for researchers, designers, and artists to consider opportunities for interactivity. While the spectrum aspires to be comprehensive, new tools and media continually reshape the interactivity landscape, and edge cases undoubtedly exist. We consider such cases to be good fodder for discussion about new forms of interactivity. Further, this research does not endeavor to describe interactivity from the perspective of the performer or to describe audience characteristics (e.g., culture, size, and location). Such perspectives may have unique characteristics that may affect interactivity.

Future work will validate the clarity, precision, and effectiveness of the spectrum by interviewing experts in a range of domains. To help practitioners learn from other domains, we plan to use our taxonomy to survey a range of audiences, 
performers, and creators who participate in interactive audience experiences, allowing designers to compare diverse interaction experiences and identify patterns that emerge across domains. This will enable designers to actively consider the novelty and practicality of their interactivity designs, identifying patterns, and anticipate challenges that may arise in experimental designs.

Acknowledgements. Thank you to Jessica Hammer and Theresa Tanenbaum for their generous feedback and support.

\section{References}

1. Allen, W.: The Kugelmass Episode. New Yorker, Braunschweig (1977)

2. Anderson, D., Gosselin, V.: Private and public memories of expo 67: a case study of recollections of montreal's world's fair, 40 years after the event. Mus. Soc. 6(1), $1-21(2008)$

3. Andersson, D., Brigante, R.: Impressive peter pan's flight interactive queue debuts dazzling pixie dust at Walt Disney World, January 2015. http://goo.gl/5hddCa

4. Azad, S., Saldanha, C., Gan, C.H., Riedl, M.O.: Mixed reality meets procedural content generation in video games. In: AAAI Conference on Artificial Intelligence and Interactive Digital Entertainment. AAAI Press (2016)

5. Azad, S., Saldanha, C., Gan, C.H., Riedl, M.O.: Procedural level generation for augmented reality games. In: Twelfth Artificial Intelligence and Interactive Digital Entertainment Conference (2016)

6. Azad, S., Xu, J., Yu, H., Li, B.: Scheduling live interactive narratives with mixedinteger linear programming. In: AAAI Conference on Artificial Intelligence and Interactive Digital Entertainment (2017)

7. Bartle, R.A.: Designing Virtual Worlds. New Riders, San Francisco (2004)

8. Bell, C.: Ritual: Perspectives and Dimensions-Revised Edition. Oxford University Press, Oxford (1997)

9. Benford, S., et al.: The frame of the game: blurring the boundary between fiction and reality in mobile experiences. In: Proceedings of the SIGCHI Conference on Human Factors in Computing Systems, pp. 427-436. ACM (2006)

10. Bernardo, F.: Music video games in live performance: catachresis or an emergent approach? In: Videojogos 2014-Conferência de Ciências E Artes Dos Videojogos (2014)

11. Boal, A.: Theater of the Oppressed. Pluto Press, London (2000)

12. Bradford, T.W., Sherry Jr., J.F.: Domesticating public space through ritual: tailgating as vestaval. J. Consum. Res. 42(1), 130-151 (2015)

13. Brignull, H., Rogers, Y.: Enticing people to interact with large public displays in public spaces. In: Proceedings of INTERACT, vol. 3, pp. 17-24 (2003)

14. Brock, T.C., Livingston, S.D.: The need for entertainment scale. In: The Psychology of Entertainment Media, pp. 259-278. Erlbaum Psych Press (2003)

15. Brockmyer, J.H., Fox, C.M., Curtiss, K.A., McBroom, E., Burkhart, K.M., Pidruzny, J.N.: The development of the game engagement questionnaire: a measure of engagement in video game-playing. J. Exp. Soc. Psychol. 45(4), 624-634 (2009)

16. Brooker, W.: Conclusion: overflow and audience. In: Brooker, W., Jermyn, D. (eds.) The Audience Studies Reader. Routledge (2003) 
17. Bukatman, S.: There's always Tomorrowland: Disney and the hypercinematic experience. October 57, 55-78 (1991)

18. Burn, A.: Potter-literacy: from book to game and back again; literature, film, game and cross-media literacy. Pap. Explor. Child. Lit. 14(2), 5-17 (2004)

19. Cavazza, M., Charles, F., Mead, S.J.: Character-based interactive storytelling. IEEE Intell. Syst. 17(4), 17-24 (2002)

20. Cavazza, M., Lugrin, J.L., Pizzi, D., Charles, F.: Madame bovary on the holodeck: immersive interactive storytelling. In: Proceedings of the 15th ACM International Conference on Multimedia, pp. 651-660. ACM (2007)

21. Cordova, D.I., Lepper, M.R.: Intrinsic motivation and the process of learning: beneficial effects of contextualization, personalization, and choice. J. Educ. Psychol. 88(4), 715 (1996)

22. Cover, R., et al.: Interactivity: reconceiving the audience in the struggle for textual 'control' of narrative and distribution. Aust. J. Commun. 31(1), 107 (2004)

23. Coverage, B.S.: In her own words: Sarah horn shares inspirational story of singing with Kristin Chenoweth at the hollywood bowl and going viral! August 2013. https://goo.gl/Afa6jE

24. Cross, G., Walton, J.K.: The Playful Crowd: Pleasure Places in the Twentieth Century. Columbia University Press, New York (2005)

25. Csikszentmihalyi, M.: Flow: the psychology of optimal performance (1990)

26. Cummings, J.J., Bailenson, J.N.: How immersive is enough? A meta-analysis of the effect of immersive technology on user presence. Med. Psychol. 19(2), 272-309 (2016)

27. DeMartino, N.: Why transmedia is catching on (part 1). Accessed 2011

28. Druin, A.: Cooperative inquiry: developing new technologies for children with children. In: Proceedings of the SIGCHI Conference on Human Factors in Computing Systems, pp. 592-599. ACM (1999)

29. Durrant, A., Kirk, D.S., Benford, S., Rodden, T.: Pursuing leisure: reflections on theme park visiting. Comput. Support. Coop. Work (CSCW) 21(1), 43-79 (2012)

30. Eicher, J.B.: Influence of changing resources on clothing-textiles and quality of life. In: Combined Proceedings, Easter, Central, and Western Regional Meetings of Association of College Professors of Textiles and Clothing. Association of College Professors of Textiles and Clothing (1981)

31. Engeser, S., Rheinberg, F.: Flow, performance and moderators of challenge-skill balance. Motiv. Emot. 32(3), 158-172 (2008)

32. Ermi, L., Mäyrä, F.: Fundamental components of the gameplay experience: analysing immersion. Worlds Play Int. Perspect. Digit. Games Res. 37(2), 37$53(2005)$

33. Everett, R.: Communication technology: the new media in society (1986)

34. Farbood, M.M., Pasztor, E., Jennings, K.: Hyperscore: a graphical sketchpad for novice composers. IEEE Comput. Graph. Appl. 24(1), 50-54 (2004)

35. Fechtmann, T.J.V.: The best queues in Walt Disney World (2015). http://blog. touringplans.com/2015/07/01/best-queues-walt-disney-world/

36. Fine, G.A.: Shared Fantasy: Role Playing Games as Social Worlds. University of Chicago Press, Chicago (2002)

37. Forlizzi, J., Battarbee, K.: Understanding experience in interactive systems. In: Proceedings of the 5th Conference on Designing Interactive Systems: Processes, Practices, Methods, and Techniques, DIS 2004, pp. 261-268. ACM, New York (2004). https://doi.org/10.1145/1013115.1013152 
38. Freeman, J.: Extreme sight-reading, mediated expression, and audience participation: Real-time music notation in live performance. Comput. Music J. 32(3), 25-41 (2008)

39. Fron, J., Fullerton, T., Morie, J.F., Pearce, C.: Playing dress-up: costumes, roleplay and imagination. Philos. Comput. Games, 24-27 (2007)

40. Gahr, S.: The art of dance, February 2017. https://www.warhol.org/the-art-ofdance/

41. Garris, R., Ahlers, R., Driskell, J.E.: Games, motivation, and learning: a research and practice model. Simul. Gaming 33(4), 441-467 (2002)

42. Gilbert, L., Moore, D.R.: Building interactivity into web courses: tools for social and instructional interaction. Educ. Technol. 38(3), 29-35 (1998)

43. Gilroy, S., Porteous, J., Charles, F., Cavazza, M.: Exploring passive user interaction for adaptive narratives. In: Proceedings of the 2012 ACM International Conference on Intelligent User Interfaces, pp. 119-128. ACM (2012)

44. Giovagnoli, M.: Transmedia Storytelling: Imagery, Shapes and Techniques. Etc Press (2011)

45. Goertz, L.: Wie interaktiv sind Medien? na (1995)

46. Goffman, E.: Interaction Ritual: Essays in Face to Face Behavior. Aldine Transaction, Piscataway (2005)

47. Goldman, A.: Predicting and motivating achievement in self-paced learning: a formative design, study and evaluation. Ph.D. thesis, University of Maryland, College Park (2014)

48. Green, M.C., Brock, T.C., Kaufman, G.F.: Understanding media enjoyment: the role of transportation into narrative worlds. Commun. Theor. 14(4), 311-327 (2004)

49. Green, M.C., Garst, J., Brock, T.C.: The power of fiction: determinants and boundaries. Psychol. Entertainment Media Blurring Lines Between Entertainment Persuasion, 161-176 (2004)

50. Guha, M.L., Druin, A., Fails, J.A.: Cooperative inquiry revisited: reflections of the past and guidelines for the future of intergenerational co-design. Int. J. ChildComput. Interac. 1(1), 14-23 (2013)

51. Gupta, R., Shah, P., George, L., Pramer, E.: Harry Pottar. http://etv.gatech. edu/2016/05/16/harry-pottar-2/

52. Hoffman, E., Slotnick, S.: Design for the 21st century: media lab style. Des. Manag. Rev. 26(1), 32-39 (2015)

53. Ito, M.: Intertextual enterprises: writing alternative places and meanings in the media mixed networks of Yugioh. ET Cult. Anthropol. Outerspaces, 180-199 (2005)

54. Jacob, M., Magerko, B.: Viewpoints AI. In: Proceedings of the 2015 ACM SIGCHI Conference on Creativity and Cognition, pp. 361-362. ACM (2015)

55. Jensen, J.F.: Interactivity: tracking a new concept in media and communication studies. Nordicom Rev. 12(1) (1998)

56. Kershaw, B.: Oh for unruly audiences! Or, patterns of participation. In: Twentieth-century Theatre. Modern Drama XLIV.2, pp. 133-154 (2001)

57. Khut, G.P.: Interactive art as embodied inquiry: working with audience experience. In: Engage: Interaction, Art and Audience Experience (2007)

58. Kiili, K., Lainema, T., de Freitas, S., Arnab, S.: Flow framework for analyzing the quality of educational games. Entertainment Comput. 5(4), 367-377 (2014)

59. Kronzek, A.Z., Kronzek, E.: The Sorcerer's Companion: A Guide to the Magical World of Harry Potter. Broadway (2010) 
60. Lancaster, K.: When spectators become performers: contemporary performanceentertainments meet the needs of an "unsettled" audience. J. Popular Cult. 30(4), 75-88 (1997)

61. Laurel, B.: Interface as mimesis, In: Norman, D.A., Draper, S.W. (eds.) User Centred System Design: New Perspectives on Human-computer Interaction (1986)

62. Laurel, B.: Computers as Theatre. Addison-Wesley, Reading (1991)

63. Laurel, B.: Interface agents: metaphors with character. In: Human Values and the Design of Computer Technology, pp. 207-219 (1997)

64. Lindley, C.: The semiotics of time structure in ludic space as a foundation for analysis and design. Game Stud. 5(1), 2005 (2005)

65. Long, D., McKlin, T., Weisling, A., Martin, W., Guthrie, H., Magerko, B.: Trajectories of physical engagement and expression in a co-creative museum installation. In: Proceedings of the 2019 on Creativity and Cognition, pp. 246-257. ACM (2019)

66. Lv, Z., Halawani, A., Feng, S., Ur Réhman, S., Li, H.: Touch-less interactive augmented reality game on vision-based wearable device. Pers. Ubiquit. Comput. 19(3-4), 551-567 (2015)

67. Machado, I., Martinho, C., Paiva, A.: Once upon a time. In: Published in Fall Symposium on Narrative Intelligence of AAAI (1999)

68. Marshall, M., Fiore, Q., Agel, J.: The Medium is the Massage: An Inventory of Effects (1967)

69. Mateas, M., Stern, A.: Façade: an experiment in building a fully-realized interactive drama. In: Game Developers Conference, vol. 2 (2003)

70. Mateas, M., Stern, A.: Interaction and narrative. Game Des. Reader Rules Play Anthol. 1, 642-669 (2006)

71. McAllister, G., Alcorn, M., Strain, P.: Interactive performance with wireless PDAs. In: International Computer Music Conference (2004)

72. McCool, S.F., Moisey, R.N., Nickerson, N.P.: What should tourism sustain? The disconnect with industry perceptions of useful indicators. J. Travel Res. 40(2), 124-131 (2001)

73. McDuff, D., El Kaliouby, R., Picard, R.W.: Crowdsourcing facial responses to online videos. In: 2015 International Conference on Affective Computing and Intelligent Interaction (ACII), pp. 512-518. IEEE (2015)

74. McMahan, A.: Immersion, engagement and presence. Video Game Theor. Reader 67, $86(2003)$

75. McMillan, S.J.: A four-part model of cyber-interactivity: some cyber-places are more interactive than others. New Media Soc. 4(2), 271-291 (2002)

76. Medianet: Teambuilding through improvisation. http://medianet-ny.com/ TeamBuilding.pdf

77. Michelis, D., Müller, J.: The audience funnel: observations of gesture based interaction with multiple large displays in a city center. Int. J. Hum.-Comput. Interac. $\mathbf{2 7}(6)$, 562-579 (2011)

78. Miller, J.H., Lentricchia, F., McLaughlin, T.: Critical Terms for Literary Study. University of Chicago Press, Chicago (1990)

79. Milman, A.: 13 theme park tourism and management strategy. In: Tourism Management: Analysis, Behaviour, and Strategy, p. 218 (2007)

80. Monson, I.T.: Forced migration, asymmetrical power relations and africanamerican music: reformulation of cultural meaning and musical form. World Music 32(3), 22-47 (1990)

81. Murray, J.H.: Hamlet on the Holodeck: The Future of Narrative in Cyberspace. MIT Press, Cambridge (2017) 
82. Nelson, T.J.: Sacrifice of praise: emotion and collective participation in an AfricanAmerican worship service. Sociol. Relig. 57(4), 379-396 (1996)

83. Nettl, B., Russell, M.: In the Course of Performance: Studies in the World of Musical Improvisation. University of Chicago Press, Chicago (1998)

84. O’Neill, B., Piplica, A., Fuller, D., Magerko, B.: A knowledge-based framework for the collaborative improvisation of scene introductions. In: Si, M., Thue, D., André, E., Lester, J.C., Tanenbaum, J., Zammitto, V. (eds.) ICIDS 2011. LNCS, vol. 7069, pp. 85-96. Springer, Heidelberg (2011). https://doi.org/10.1007/9783-642-25289-1_10

85. Palmer, C.T., Coe, K.: Parenting, courtship, disneyland and the human brain. Int. J. Tourism Anthropol. 1(1), 1-14 (2010)

86. Pavlik, J.V., Bridges, F.: The emergence of Augmented Reality (AR) as a storytelling medium in journalism. Journalism Commun. Monogr. 15(1), 4-59 (2013)

87. Peltonen, P., et al.: It's mine, don't touch! Interactions at a large multi-touch display in a city centre. In: Proceedings of the SIGCHI Conference on Human Factors in Computing Systems, pp. 1285-1294. ACM (2008)

88. Pohjola, M.: Autonomous Identities: Immersion as a Tool for Exploring. Empowering and (2004)

89. Pointer, M.: Charles Dickens on the Screen: The Film, Television, and Video Adaptations. Scarecrow Press, Lanham (1996)

90. Rafaeli, S.: From new media to communication. Sage Annu. Rev. Commun. Res. Advancing Commun. Sci. 16, 110-134 (1988)

91. Rand, A.: The Night of January 16th. Penguin, London (1971)

92. Reeves, S., Benford, S., O'Malley, C., Fraser, M.: Designing the spectator experience. In: Proceedings of the SIGCHI Conference on Human Factors in Computing Systems, pp. 741-750. ACM (2005)

93. Rickman, B.: The Dr. k-project. In: Advances in Consciousness Research, vol. 46, pp. 131-142 (2002)

94. Salen, K., Zimmerman, E.: Rules of Play: Game Design Fundamentals. MIT Press, Cambridge (2004)

95. Schell, J., Shochet, J.: Designing interactive theme park rides lessons learned creating Disney's Pirates of the Caribbean-Battle for the Buccaneer Gold. In: Proceedings of the 2001 Game Developers Conference, pp. 723-731 (2001)

96. Schmitt, N.C.: Casting the audience. TDR (1988-) 37(4), 143-156 (1993)

97. Shaker, N., Yannakakis, G.N., Togelius, J.: Towards automatic personalized content generation for platform games. In: AIIDE (2010)

98. Shani, A., Pizam, A.: The role of animal-based attractions in ecological sustainability: current issues and controversies. Worldwide Hospitality Tourism Themes 2(3), 281-298 (2010)

99. Shernoff, D.J., Csikszentmihalyi, M.: Cultivating engaged learners and optimal learning environments. In: Handbook of Positive Psychology in Schools, pp. 131145 (2009)

100. Shipman III, F.M.: Blending the real and virtual: activity and spectatorship in fantasy sports. In: Proceedings from DAC 2001: The Conference on Digital Arts and Culture (2001)

101. Shneiderman, B.: Designing the User Interface: Strategies for Effective HumanComputer Interaction. Pearson Education India, Bengaluru (2010)

102. Simkins, D.: The Arts of Larp: Design, Literacy, Learning and Community in Live-action Role Play. McFarland, Jefferson (2015)

103. Sit, J.K., Birch, D.: Entertainment events in shopping malls-profiling passive and active participation behaviors. J. Consum. Behav. 13(6), 383-392 (2014) 
104. Smuts, A.: Are video games art? Contemp. Aesthetics 3, 6 (2005)

105. Squinkifer, D.: Coffee! A misunderstanding (2014). http://squinky.me/mygames/coffee-a-misunderstanding/

106. Stapleton, C.B., Hughes, C.E.: Mixed reality and experiential movie trailers: combining emotions and immersion to innovate entertainment marketing. In: Proceedings of 2005 International Conference on Human-Computer Interface Advances in Modeling and Simulation (SIMCHI 2005), pp. 23-27. Citeseer (2005)

107. Steuer, J.: Defining virtual reality: dimensions determining telepresence. J. Commun. 42(4), 73-93 (1992)

108. Striner, A., McNally, B.: Transitioning between audience and performer: codesigning interactive music performances with children. In: Proceedings of the 2017 CHI Conference Extended Abstracts on Human Factors in Computing Systems, CHI EA 2017, pp. 2115-2122. ACM, New York (2017). http://doi.acm.org/ $10.1145 / 3027063.3053171$

109. Strutner, S.: This guy quit his job to play piano around the world in a beautifully unplugged life, July 2015. http://www.huffingtonpost.com/entry/dotan-negrinpiano-around-the-world_us_559fd8c0e4b096729155ecf8

110. Summerville, A., Guzdial, M., Mateas, M., Riedl, M.: Learning player tailored content from observation: platformer level generation from video traces using LSTMs. In: AAAI Conference on Artificial Intelligence and Interactive Digital Entertainment (2016)

111. Sylvester, T.: Designing Games: A Guide to Engineering Experiences. O'Reilly Media, Inc., Newton (2013)

112. Taylor, T.L., Witkowski, E.: This is how we play it: what a mega-LAN can teach us about games. In: Proceedings of the Fifth International Conference on the Foundations of Digital Games, FDG 2010, pp. 195-202. ACM, New York (2010). https://doi.org/10.1145/1822348.1822374

113. Togelius, J., Yannakakis, G.N., Stanley, K.O., Browne, C.: Search-based procedural content generation: a taxonomy and survey. IEEE Trans. Comput. Intell. AI Games 3(3), 172-186 (2011)

114. Toups, Z.O., Hammer, J., Hamilton, W.A., Jarrah, A., Graves, W., Garretson, O.: A framework for cooperative communication game mechanics from grounded theory. In: Proceedings of the First ACM SIGCHI Annual Symposium on ComputerHuman Interaction in Play, pp. 257-266. ACM (2014)

115. van Troyer, A.: Constellation: a tool for creative dialog between audience and composer. In: 10th International Symposium on Computer Music Multidisciplinary Research (2013)

116. van Troyer, A.: Enhancing site-specific theatre experience with remote partners in sleep no more. In: Proceedings of the 2013 ACM International Workshop on Immersive Media Experiences, ImmersiveMe 2013, pp. 17-20. ACM, New York (2013). https://doi.org/10.1145/2512142.2512150

117. Unger, P., Forsberg, K., Jacobsen, J.H.: Photovote: olympic judging system. In: Extended Abstracts on Human Factors in Computing Systems, CHI 2004, pp. 1670-1674. ACM, New York (2004). https://doi.org/10.1145/985921.986184

118. Van Maanen, J., Frost, P., Moore, P., Lundberg, C., Louis, M., Martin, J.: The smile factory. In: Sociology: Exploring the Architecture of Everyday Life Readings. Pine Forge Press, Newbury Park (1991)

119. Vermeulen, I.E., Roth, C., Vorderer, P., Klimmt, C.: Measuring user responses to interactive stories: towards a standardized assessment tool. In: Aylett, R., Lim, M.Y., Louchart, S., Petta, P., Riedl, M. (eds.) ICIDS 2010. LNCS, vol. 6432, pp. 38-43. Springer, Heidelberg (2010). https://doi.org/10.1007/978-3-642-16638-9_7 
120. Vorderer, P., Knobloch, S., Schramm, H.: Does entertainment suffer from interactivity? The impact of watching an interactive tv movie on viewers' experience of entertainment. Media Psychol. 3(4), 343-363 (2001)

121. Wardrip-Fruin, N., Harrigan, P.: First Person: New Media as Story, Performance, and Game. MIT Press, Cambridge (2004)

122. Whitacre, E.: A virtual choir 2,000 voices strong. https://www.ted.com/talks/ eric_whitacre_a_virtual_choir_2_000_voices_strong

123. Winkler, T.: Composing Interactive Music: Techniques and Ideas Using Max. MIT Press, Cambridge (2001)

124. Yee, N.: Facets: 5 motivation factors for why people play MMORPG's. Terra Incognita 1, 1708-1723 (2002)

125. Zeltzer, D.: Autonomy, interaction, and presence. Presence Teleoperators Virtual Environ. 1(1), 127-132 (1992)

126. Zimmerman, E.: Narrative, interactivity, play, and games: four naughty concepts in need of discipline (2004) 